\title{
Spinning-up: the case of the symbiotic X-ray binary 3A 1954+319
}

\author{
F. Fürst ${ }^{* a}$, D. M. Marcu ${ }^{b}$, K. Pottschmidt ${ }^{b}$, V. Grinberg ${ }^{a}$, J. Wilms ${ }^{a}$, and M. Cadolle Bel ${ }^{c}$ \\ ${ }^{a}$ Dr. Karl Remeis-Sternwarte E ECAP, University Erlangen-Nuremberg, Bamberg, Germany \\ ${ }^{b}$ CRESST/NASA-GSFC, Greenbelt, MD, USA E UMBC, Baltimore, MD, USA \\ ${ }^{c}$ ESAC, Madrid, Spain
}

\begin{abstract}
We present a timing and spectral analysis of the variable X-ray source 3A 1954+319. Our analysis is mainly based on an outburst serendipitously observed during INTEGRAL Key Program observations of the Cygnus region in 2008 fall and on the Swift/BAT longterm light curve. Previous observations, though sparse, have identified the source to be one of only nine known symbiotic $\mathrm{X}$-ray binaries, i.e., systems composed of an accreting neutron star orbiting in a highly inhomogeneous medium around an M-giant companion. The spectrum of 3A 1954+319 above $20 \mathrm{keV}$ can be best described by a broken power law model. The extremely long pulse period of $\sim 5.3$ hours is clearly visible in the INTEGRAL/ISGRI light curve and confirmed through an epoch folding period search. Furthermore, the light curve allows us to determine a very strong spin up of $-2 \times 10^{4} \mathrm{~h} / \mathrm{h}$ during the outburst. This spin up is confirmed by the pulse period evolution calculated from $S$ wift/BAT data. The $S$ wift/BAT data also show a long spin-down trend prior to the 2008 outburst, which is confirmed in archival INTEGRAL/ISGRI data. We discuss possible accretion models and geometries allowing for the transfer of such large amounts of angular momentum and investigate the harder spectrum of this outburst compared to previously published results.
\end{abstract}

8th INTEGRAL Workshop "The Restless Gamma-ray Universe"- Integral2010, September 27-30, 2010

Dublin Ireland

${ }^{*}$ Speaker. 


\section{Introduction}

3A 1954+319 belongs to a small class of Low Mass X-ray Binaries (LMXB), in which a neutron star orbits around an M-type companion [6]. The orbits are typically wide, making accretion via common Roche-Lobe overflow impossible. Instead, material from the wind of the companion is accreted onto the neutron star. This accretion mechanism is very common among High Mass X-ray Binaries and their O- and B-type companions with strong stellar winds, but not well investigated for late type donors. In the case of 3A 1954+319 the accretion stream onto the neutron star seems to be highly variable as irregular X-ray flares are observed as well as times where the source is hardly detected at all. Binaries hosting a neutron star and an M-type companion are commonly referred to as Symbiotic X-ray Binaries (SyXB), in analogy of Symbiotic Stars consisting of a White Dwarf and a late-type star. These systems were named after their optical spectra in which both components, the hot White Dwarf and the cold companion are seen. Such features are not seen in $\mathrm{SyXB}$, as the neutron star is invisible in the optical. A close look in X-rays is thus indispensable for a description of those systems.

Only 7 sources are clearly confirmed as SyXBs today [9], two more are under discussion. Only very few of the SyXB, like GX $1+4$ or $4 \mathrm{U} 1700+24$, have already been studied in detail with different $\mathrm{X}$-ray instruments, including high-resolution CCD detectors, and much is still to be learned about these systems and the properties of their wind and accretion mechanism. Being easily observable in X-rays, SyXB provide a unique opportunity for studying the wind properties of late type stars, via X-ray variations of the emission lines and absoprtion column.

3A 1954+319 was detected in the late 1970s with Uhuru and Ariel V [3, 12]. From the analysis of Ginga data it was proposed that the system could be a HMXB [11]. No further detailed studies were performed until the companion was identified as an M-star using an accurate Chandra-position, placing the system in the group of SyXB [6]. Soon thereafter a $\sim 5 \mathrm{~h}$ period was discovered in $S w i f t / B A T$ data [6]. This led to closer investigations, and the analysis of the broadband spectrum of 3A 1954+319, using INTEGRAL and BeppoSAX [8]. Further spectral analysis showed that the spectrum is consistent with the typical spectrum expected from an accreting neutron star and can be modelled using an either a cutoff powerlaw model or a thermal Comptonization model [7].

A strong decrease of the pulse period from $5.17 \mathrm{~h}$ to $5.09 \mathrm{~h}$ during an outburst in 2004/2005 and possible physical explanations thereof was reported in Swift/BAT data [2]. The period itself is most likely the spin period of the neutron star, as it is much too short for the expected wide orbit of the system and no other significant periods have been found between $5 \times 10^{-3}-3.5 \times 10^{+8} \mathrm{~s}[2,7]$. It is also unlikely that the observed decrease in the period is due to the orbit of the binary system, as the mass function would then lead to impossible values of the mass of the companion, assuming a canonical mass of $1.4 \mathrm{M}_{\odot}$ for the neutron star.

In 2008 November INTEGRAL serendipitously observed a strong outburst of 3A 1954+319, obtaining high quality lightcurves and spectra, which are presented here.

\section{Lightcurves and Timing}

The long-term daily lightcurve of 3A $1954+319$ as measured with $S$ wift/BAT is shown in 

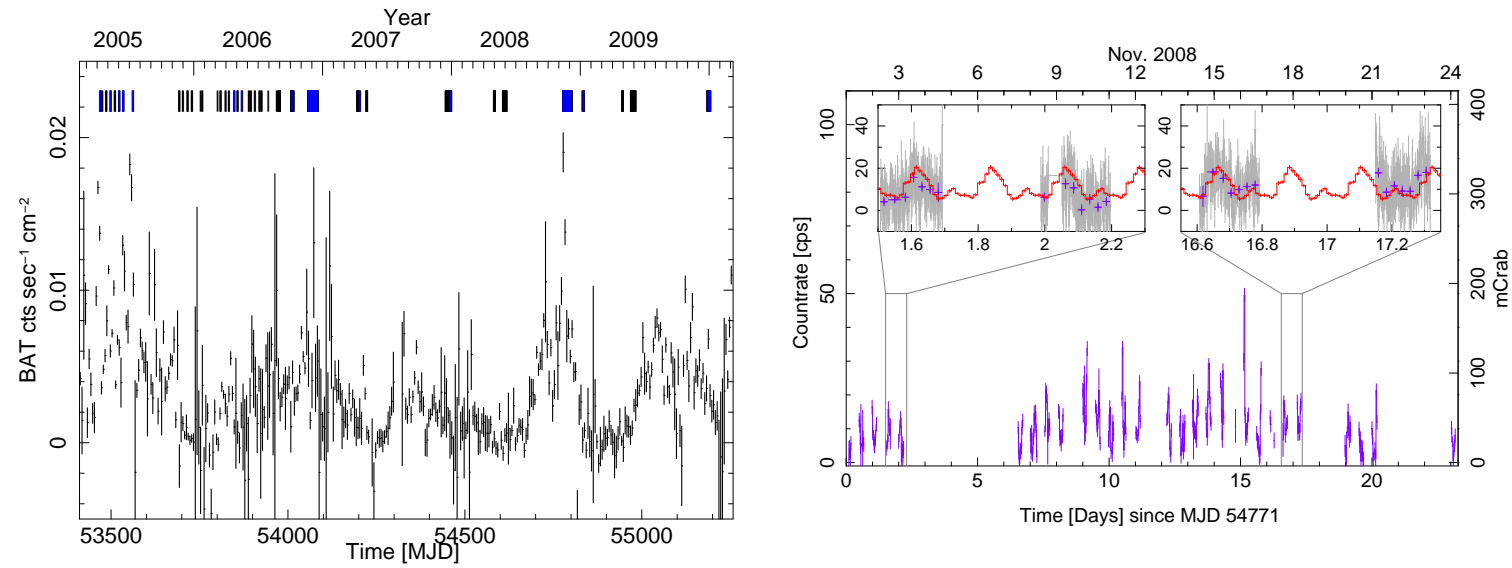

Figure 1: Left: Lightcurve as measured with $S$ wift/BAT. The tickmarks above indicate times of INTEGRAL observations, with the blue tickmarks showing times when the source was significantly detected in INTEGRAL/ISGRI. Right: INTEGRAL/ISGRI 20-100 keV lightcurve in November 2008. The data were binned to Science Window resolution. The insets show zooms and a more detailed lightcurve with $100 \mathrm{~s}$ resolution. Overplotted in red is the average pulse profile.

Fig. 1 (left). It is clearly seen that 3A $1954+319$ shows irregular flaring with some flares being as bright as almost $100 \mathrm{mCrab}$ and other episodes where it falls below the detection limit of Swift/BAT. Tickmarks above the lightcurve mark times when 3A 1954+319 was in the field of view of INTEGRAL/ISGRI. A relatively dense sampling of INTEGRAL observations was performed during a flare in 2008 November when Key Programme observations of Cygnus X-1 (PI Wilms) were scheduled. As seen in the left panel of Fig. 1 this flare was one of the brightest since the monitoring of Swift/BAT, with an average flux over the outburst of $\sim 8.6 \times 10^{-10} \mathrm{erg} \mathrm{cm}^{-2} \mathrm{~s}^{-1}$ between $20-100 \mathrm{keV}$, i.e., $\sim 60 \mathrm{mCrab}$ and a peak flux of more than $180 \mathrm{mCrab}$. We extracted $I N$ TEGRAL data of that outburst using the standard Off-line Science Analysis (OSA) 8.0 to obtain spectra and using ii_light, as distributed with OSA 7.0, to obtain lightcurves with a time resolution of $100 \mathrm{~s}$. The INTEGRAL/ISGRI lightcurve of that period is shown in Fig. 1 (right). Even though 3A 1954+319 was in an overall bright state during that time the INTEGRAL data show that the X-ray flux is still highly variable and varies by a factor of $\sim 20$. Using the epoch-folding technique [5], a period of $\sim 5.3 \mathrm{~h}$ is clearly detected in the data. Compared to earlier results [2], the pulse period is significantly longer, i.e., the neutron star has spun down between 2005 and 2008.

As a strong spin up trend was found in the 2005 flare [2] , we split the INTEGRAL lightcurve into three equally long parts and searched in each one for the pulse period separately. We found a clear spin up trend in these data, with the pulse period decreasing from $5.34 \mathrm{~h}$ in the beginning of the outburst to $5.26 \mathrm{~h}$ in the end. The results of the epoch-folding are shown in Fig. 2. From this analysis we determined the pulse ephemeris to be (during that outburst): $T_{0}^{\mathrm{MJD}}=54782.6897 \mathrm{~d}$ (mean of observation), $P=5.3085 \mathrm{~h}$, and $\dot{P}=-2 \times 10^{-4} \mathrm{~h} / \mathrm{h}$. To check for consistency, we folded the lightcurve with this ephemeris to obtain a pulse profile and compared it to the measured lightcurve. As seen in the insets of the right panel of Fig. 1 the pulse profile describes the lightcurve very well, in the beginning of the outburst as well as close to the end. This is also the first time that the pulsed flux of 3A 1954+319 is directly visible in a lightcurve, showing that 3A 1954+319 does not show large pulse-to-pulse variations. 

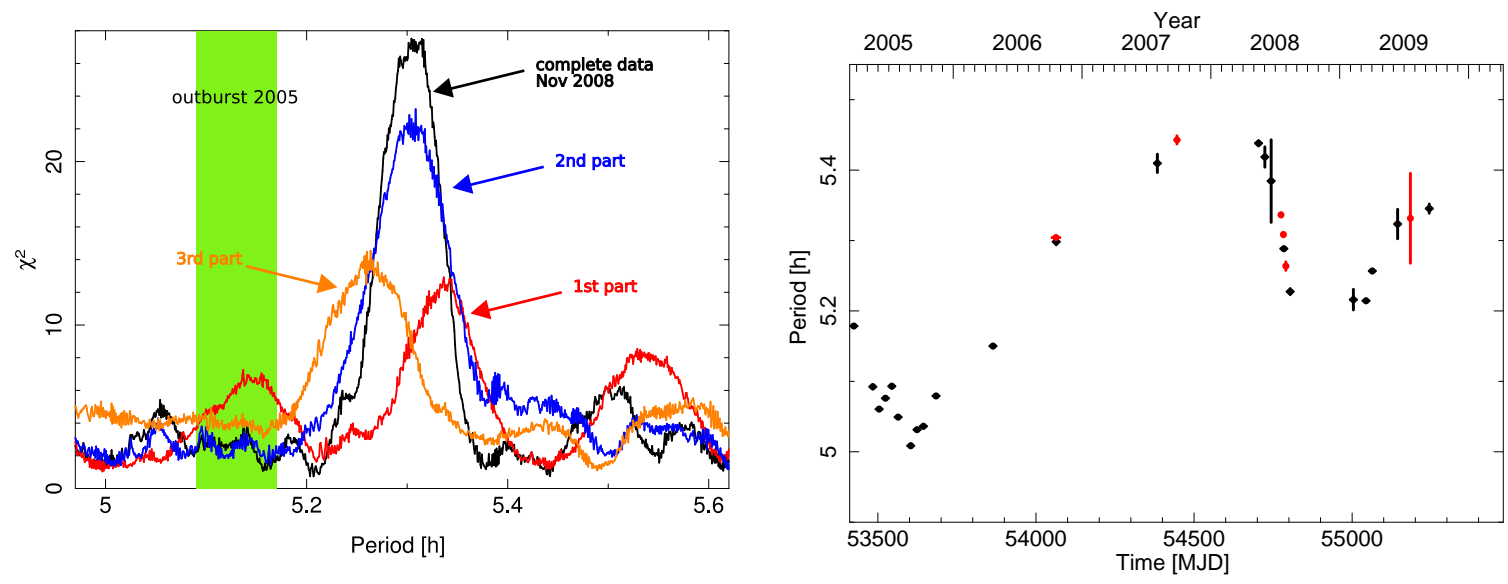

Figure 2: Left: Results of the epoch folding analysis, with the lightcurve separated into three equally long parts. Additionally the overall result is shown in black and the range of the 2005 pulse period is marked in green. Right: Long term pulse period evolution, measured with Swift/BAT (black) and INTEGRAL/ISGRI (red).

In order to put our INTEGRAL results into perspective, we updated the $S$ wift/BAT period analysis of 3A 1954+319, again using epoch-folding. Using all publicly available $S$ wift/BAT lightcurve data, local period determinations were performed for $20 \mathrm{~d}$ long segments. Figure 2 shows all successful $S$ wift/BAT period measurements, that is, all data in which a period could be significantly measured. As the $S$ wift/BAT data are impaired by a low signal-to-noise ratio, we also analyzed more INTEGRAL/ISGRI archive data. Additionally to the three data points from the 2008 November outburst we could obtain 3 more measurements of the pulse period during times with extensive coverage of 3A 1954+319 with INTEGRAL. All data points, Swift/BAT and INTEGRAL/ISGRI, are plotted together in the right panel of Fig. 2 and the measurements for both satellites agree very well. The pulse period is slowly decreasing during quiescence but increases strongly during prominent outbursts, as expected from standard accretion theory [4]. One such outburst was measured in 2005 [2], the other one was caught serendipitously by INTEGRAL in 2008 November and reported here for the first time. Even though in between these outbursts 3A 1954+319 shows smaller flares, no measurable spin up is seen. Correlating the luminosity with the change in pulse period led to no conclusive result, however, this may be due to the sparse sampling of the pulse period between outbursts.

\section{Spectral analysis}

The INTEGRAL observations present the unique possibility to gain knowledge about the accretion physics during a strong flare of 3A 1954+319, which was accompanied by a strong spin up of the neutron star. As! the source is close to INTEGRAL/ISGRI's detection limit during pulse minimum, we created a peak spectrum of only those $50 \%$ of data, which were measured at phases close to the major peak of the pulse profile, thereby improving the signal-to-noise ratio. Comparing pulse profiles at different energies above $20 \mathrm{keV}$ did not reveal any strong energy dependence with pulse phase, indicating that the spectrum is not sensitive to the selected pulse phase and that the obtained spectrum is very similar to an overall phase averaged spectrum. This filtering resulted 


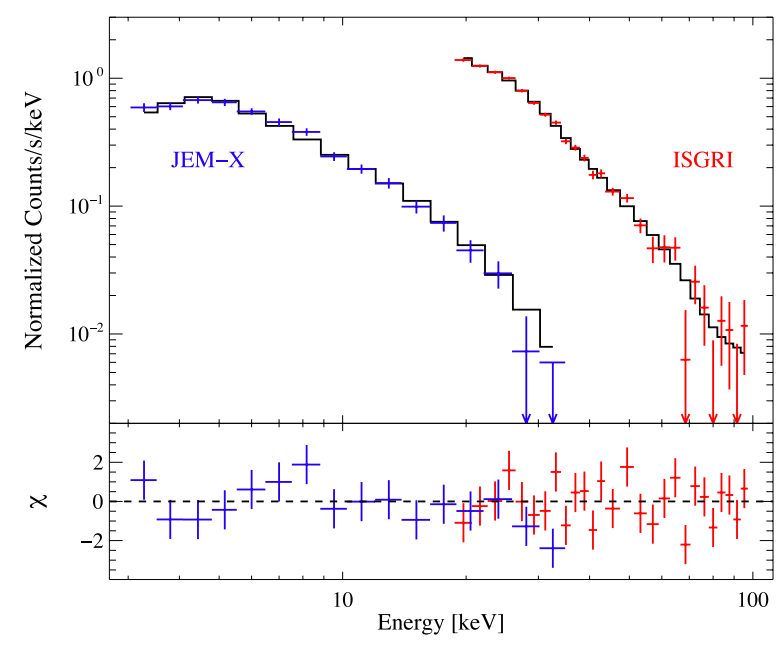

Figure 3: Top: Spectrum taken with JEM-X and ISGRI, together with the best fit model. Bottom: Residuals of the best fit.

Table 1: Best fit parameters of the bknpower model.

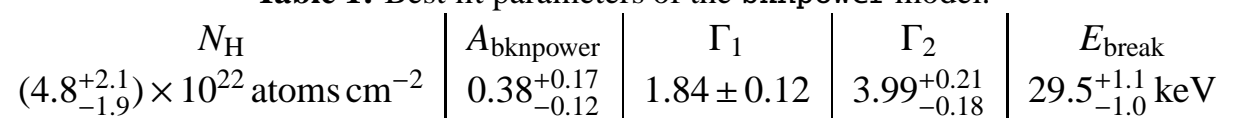

in the selection of 75 Science Windows ( $\mathrm{ScWs)} \mathrm{in} \mathrm{ISGRI,} \mathrm{with} \mathrm{the} \mathrm{additional} \mathrm{restriction} \mathrm{that} \mathrm{the}$ pointing offset was $<10^{\circ}$ to $3 \mathrm{~A} 1954+319$, leading to a pulse peak spectrum with $88 \mathrm{ks}$ exposure in ISGRI and $22 \mathrm{ks}$ in JEM-X. Modelling these JEM-X and ISGRI data between 3-100 keV with the published models lead to unacceptable descriptions only. A much better description in terms of $\chi_{\text {red }}^{2}$ ( $\chi^{2}=43.91$ with 37 d.o.f.) was obtained, when using the bknpower model, a model consisting of two power-laws with different photon indices $\Gamma_{1}$ and $\Gamma_{2}$, with the change from one photon index to the other taking place at a specific break energy $E_{\text {break }}$. It was not necessary to apply systematics for this fit. The model was attenuated by photo absorption at lower energies, expressed as equivalent hydrogen column density $N_{\mathrm{H}}$. Data and the best fit model are shown in Fig. 3, and the parameters of the best fit model can be found in Tab. 1. The required new description of the model compared to the older spectra leads to an overall harder spectrum, as no exponential cut-off is visible. The soft energy continuum has a slope which is consistent with previous published results, see, e.g., [8].

\section{Outlook \& Conclusions}

We presented a timing and spectral analysis of INTEGRAL data of the large outburst of 3A 1954+319 in 2008 November. We confirm the unusually long spin period and measure a value of $\sim 5.3 \mathrm{~h}$. During the outburst we found that the pulse period is drastically increasing, with a spin up rate as high as $\dot{P}=-2 \times 10^{-4} \mathrm{~h} / \mathrm{h}$. This rate is a magnitude higher than the one found for the 2005 outburst [2]. As these authors already stated, a change of this magnitude can not be due to the orbital motion, as it would lead to a mass-function for the companion of $>5 \times 10^{6} \mathrm{M}_{\odot}$. On the other hand, if we assume that the neutron star is spinning close to equilibrium, i.e., that the Alfvén radius is equal to the Keplerian co-rotating radius, then a magnetic field of $\sim 10^{15} \mathrm{G}$ would be required. 
In the standard accretion picture, this assumption is justified as only close to equilibrium the torque can easily change direction [4]. However, more sophisticated accretion models have been proposed for other sources, including retrograde spinning accretion disks [10] and spherical wind accretion. If no stable accretion disk forms and material and momentum is accreted from the stellar wind, a torque transfer of this magnitude might be possible (see Postnov et al. elsewhere in these proceedings). Continued monitoring of 3A 1954+319 is necessary in any case to clarify possible models and to monitor the pulse period evolution.

The pulse peak spectrum of 3A $1954+319$ we presented is distinctly harder than the previously published spectra, but is otherwise featureless. Especially, we find no indication for a Cyclotron Resonant Scattering Feature (CRSF), as seen in many other X-ray binaries, where the neutron star possesses a magnetic field $\geq 10^{12} \mathrm{G}$. Such a feature is the only direct way to measure the magnetic field strength in the X-ray producing region and could clarify the size of the accretion region. If 3A $1954+319$ really has a magnetic field on the order of $10^{15} \mathrm{G}$, it is not suprising that we do not see any CRSF, as the fundamental line energy would be $\geq 10 \mathrm{MeV}$, where the source is not visible anymore. For a more detailed presentation of the results see a forthcoming publication by Marcu et al., 2011, in prep.

Acknowledgements: This work was funded by the BMWi under DLR grant 50 OR 0808 and supoorted via a DAAD fellowship.

[1] R. Corbet, L. Barbier, S. Barthelmy, J. Cummings, E. Fenimore, N. Gehrels, D. Hullinger, H. Krimm, C. Markwardt, D. Palmer, A. Parsons, T. Sakamoto, G. Sato, and J. Tueller, Swift/BAT Detection of a 5 hour Period in $4 U$ 1954+31, ATel 797 (2006).

[2] R. H. D. Corbet, J. L. Sokoloski, K. Mukai, C. B. Markwardt, and J. Tueller, A comparison of the variability of the symbiotic $X$-Ray binaries $G X$ $1+4,4 U 1954+31$, and $4 U 1700+24$ from Swift/BAT and RXTE/ASM observations, ApJ 675, 1424-1435 (2008).

[3] W. Forman, C. Jones, L. Cominsky, P. Julien, S. Murray, G. Peters, H. Tananbaum, and R. Giacconi, The fourth Uhuru catalog of X-ray sources., A\&AS 38, 357-412 (1978).

[4] P. Ghosh and F. K. Lamb, Accretion by rotating magnetic neutron stars. III - accretion torques and period changes in pulsating $x$-ray sources, ApJ 234, 296-316 (1979).

[5] D. A. Leahy, R. F. Elsner, and M. C. Weisskopf, On searches for periodic pulsed emission - The Rayleigh test compared to epoch folding, ApJ 272, 256-258 (1983).

[6] N. Masetti, M. Orlandini, E. Palazzi, L. Amati, and F. Frontera, M-type giants as optical counterparts of $X$-ray sources $4 U 1700+24$ and $4 U$ 1954+319, A\&A 453, 295-299 (2006).
[7] N. Masetti, E. Rigon, E. Maiorano, G. Cusumano, E. Palazzi, M. Orlandini, L. Amati, and F. Frontera, $X$-ray broad-band study of the symbiotic $x$-ray binary $4 U$ 1954+31, A\&A 464, 277-278 (2007).

[8] F. Mattana, D. Götz, M. Falanga, F. Senziani, A. de Luca, P. Esposito, and P. A. Caraveo, A new symbiotic low mass $X$-ray binary system: $4 U$ 1954+319, A\&A 460, L1-L4 (2006).

[9] E. Nespoli, J. Fabregat, and R. E. Mennickent, $K$-band spectroscopy of IGR J16358-4726 and IGR J16393-4643: two new symbiotic X-ray binaries, A\&A, 516, A94 (2010).

[10] Rosalba Perna, Enrico Bozzo, and Luigi Stella, On the Spin-up/Spin-down transitions in accreting $X$-Ray binaries, ApJ 639, 363-376 (2006).

[11] R. W. Tweedy, R. S. Warwick, and R. Remillard, The 3A1954+319: a possible supergiant X-ray binary in proceedings of Two Topics in X-ray Astronomy: Vol. 1 (ESA-SP-296), (J. Hunt and B. Battrick, eds.), November 1989, pp. 661-663.

[12] R. S. Warwick, N. Marshall, G. W. Fraser, M. G. Watson, A. Lawrence, C. G. Page, K. A. Pounds, M. J. Ricketts, M. R. Sims, and A. Smith, The ariel $V(3 A)$ catalogue of $X$-ray sources - I. Sources at low galactic latitude (absolute value of $b$ less than $10 \mathrm{deg}$ ), MNRAS 197, 865-891 (1981). 